\title{
A Pilot-Plant Test of a Membrane Bioreactor with a Novel Membrane Made from Chlorinated Poly (Vinyl Chloride) (CPVC) and Hydroxy-Propyl Cellulose
}

\author{
Junsuke Morita, Norifumi Shimada, Masao Higashi and Tooru Kitagawa* \\ Membrane-Structural Development Group, Research Center, Toyobo Co., Ltd. 2-1-1 Katata, Otsu, Shiga
520-0292, Japan
}

Abstract: A pilot-plant test that can treat an amount of 30 ton per day of wastewater is performed. The purpose of this test is to prove the usefulness and applicability of newly developed microfiltration membranes made from chlorinated poly (vinyl chloride) and polyester nonwoven. Here, the hydrophilicity of membranes is important and hydroxyl-propyl cellulose is used to mitigate their hydrophobicity. The membrane consists of the novel structure in which small particles made from hydroxyl-propyl cellulose gel are homogeneously dispersed and attached on the surface of micro fibrils in the structure. The result of the pilot-plant test shows that the newly developed membrane has anti-fouling properties better than that of a conventional membrane made from chlorinated poly (vinyl chloride) by another company. It still shows high hydrophilicity after the use of one year, while the conventional one loses such properties. Saving electricity in producing water is one of important issues in developing membrane bioreactor systems and the case in use of newly-developed membranes is revealed to need $2.0 \mathrm{kWh}$ of electricity to produce $1 \mathrm{~m}^{3}$ of filtered water. This amount is fairly good and reasonable when the pilot-plant test is considered to belong to a medium-size facility.

Keywords: MBR, chlorinated poly(vinyl chloride), hydroxy-propyl cellulose, fouling, hydrophilicity.

\section{INTRODUCTION}

This article concerns a pilot plant test of a membrane bioreactor (MBR) to purify sewage using a newly developed flat-sheet type micro-filtration (MF) membrane. As the properties for the membrane is reported in a separate publication, we are succeeding in developing a novel flat sheet micro-filtration membrane that has a high hydrophilic nature with open porous structures [1]. Its properties stand for a long time and will contribute low fouling properties. The membrane is made from chlorinated Poly (Vinyl Chloride) (CPVC) and polyester nonwoven. It has a long-standing hydrophilic nature with anti-fouling properties, because hydroxyl-propyl cellulose (HPC) is applied as a hydrophilic material on the surface of the membrane and cured to transform itself to an insoluble form. It is fixed with a form of fine gel particles, because the transition from solution to gel is induced irreversibly by heat as reported in the literature, so that the particles once formed are not easily soluble in water in a bioreactor [2]. It should be noted that the particles should be well dispersed, which is achieved with finely selecting the phase separation conditions. Please note that the fact of the transition of HPC to gel from solution was widely known but the detailed configuration in which it took a gel particle form on the

\footnotetext{
*Address correspondence to this author at the Membrane-Structural Development Group, Research Center, Toyobo Co., Ltd. 2-1-1 Katata, Otsu, Shiga 520-0292, Japan; Tel: +81-77-571-0034; Fax: +81-77-571-0121;

E-mail: Tooru_Kitagawa@toyobo.jp
}

surface of the membrane has not been reported before and this new fact was made clear first by the present authors. The details of its novel structures and physical properties of newly developed membranes are also shown in a separate publication [1]. The membrane has shown its good anti-fouling properties under a relatively high flux operation condition of $1.0 \mathrm{~m} / \mathrm{d}(41.7$ $\mathrm{LMH}$ ) in a laboratory scale test. However, this demonstration of the laboratory test itself is not enough to persuade the people who are interested in MBR technologies and apply them to an actual use in their plants or facilities. It is necessary for us to prove the developed membrane is working well in a real use for purifying sewage or wastewater from industry or household effluents. It is important to show potentialities of the developed membrane in a pilotplant scale test for over one year. We will plan a 30 ton per day scale test to purify sewage (wastewater) and the amount the pilot plant treats should be designed according to the demand in which it will cover all output of wastewater from a small factory.

Next, we will discuss a material for membranes. To make a membrane for MBR in general, polyethylene $(\mathrm{PE})$, polyvinyliden fluoride (PVDF), polytetrafluoroethylene (PTFE), polyether sulfone (PES) and chlorinated poly (vinyl chloride) (CPVC) are selected [3]. One of the criteria to choose the material for membrane is to see if it can give a porous structure. Other required conditions are the applicability of hydrophilic properties and anti-chemical properties. PVDF is a good choice to give a hollow fiber 
membrane with high mechanical properties when it is processed with thermal-induced phase separation, but some mitigation of its high hydrophobic properties may be an issue and should be considered [4]. PTFE has a strong anti-chemical nature and may be useful to treat strong acid or alkaline wastewater and PE has an advantage in cost. But both membranes made by drawing and may be disadvantageous in having broad pore size distribution. There might be a risk to leak sludge if the control of pore size distribution is not enough. The PES membrane is made through a phase separation process with coagulation and the surface of the membrane is physically weak against a collision of external particles in bioreactor, because it often includes a larger void structure just under the surface of the membrane [5].

This time a membrane which is made from CPVC and polyethylene terephthalate (PET) nonwoven is used (Table 1). The CPVC is useful to the MBR, because it forms a very high porous structure with open pores and has a medium resistance against chemicals. The pore size can be made small in the range of $0.1-$ $0.5 \mu \mathrm{m}$ with very narrow pore-size distribution [6-10]. This is made possible with keenly adjusting the phase separation condition of temperature, the solution concentration and the composition of solvent and nonsolvents [11]. This high-open porous structure has been already proved with microscopic methods such as scanning (SEM) and transmission electron microscopy (TEM). The novel structure having hydroxyl-propyl cellulose (HPC) gel particles dispersed homogeneously on the surface of the membrane is also shown with TEM and electron spectroscopy for chemical analysis (ESCA). In fact an improvement to keep long-term hydrophilic properties was an issue for CPVC membranes, but it was solved with a proposed membrane by the present authors. Both structural and chemical features give good anti-fouling properties and will be useful for a long-term use in the pilot scale test of MBR.

To show an applicability of our developed membrane to treat sewage, we plan a field test of a membrane bioreactor having a 30 ton per day output at Konan-Chubu Wastewater Treatment Plant (sewage facility). It is located in Yabase-Kihan Island of the west part of Kusatsu city in Shiga prefecture beside Lake Biwa (the largest inland fresh water lake in Japan). Shiga prefectural government runs the sewage facility. The prefectural government has a program to facilitate the development of technologies of local private companies or institutes in Shiga prefecture and provides an opportunity with them to develop new engineering systems or advanced materials on sewage water treatment. This field test is performed as one of such programs under the management and courtesy of the prefectural government. We can install a pilot plant machine inside the sewage facility and carry out a longrun test by the courtesy of the prefectural government, which should be well acknowledged. The plant treats the sewage of about $45 \%$ population of Shiga prefecture and outputs clean water treated in Lake Biwa.

\section{EXPERIMENTAL}

\section{Machine for the MBR Pilot-Plant Test}

A membrane bioreactor having a capacity to treat an amount of 30 ton wastewater per day was built in the site of Konan-Chubu Wastewater Treatment Plant. The photograph for the pilot plant machine is shown in Figure $\mathbf{1}$ and the block diagram is shown in Figure 2. Sewage (municipal wastewater) is taken from the initial sedimentation bath of Konan-Chubu Wastewater Treatment Plant. The membrane used for this

Table 1: Details of Specimens

\begin{tabular}{|c|c|c|c|}
\hline \multicolumn{2}{|c|}{ unit } & values \\
\hline \hline 1. Developed Membrane & \multicolumn{2}{|c|}{ CPVC + PET nonwoven } \\
\hline Material & Average & $\mu \mathrm{m}$ & 0.30 \\
\hline Pore Size & Maximum & $\mathrm{Lm} / \mathrm{m}^{2} / \mathrm{hr} / \mathrm{kPa}$ & 0.65 \\
\hline Flux & & $\mathrm{MPa}$ & 0.155 \\
\hline Bubble Point & & & \\
\hline 2. Commercial membrane purchased from another company & CPVC + PET nonwoven \\
\hline
\end{tabular}




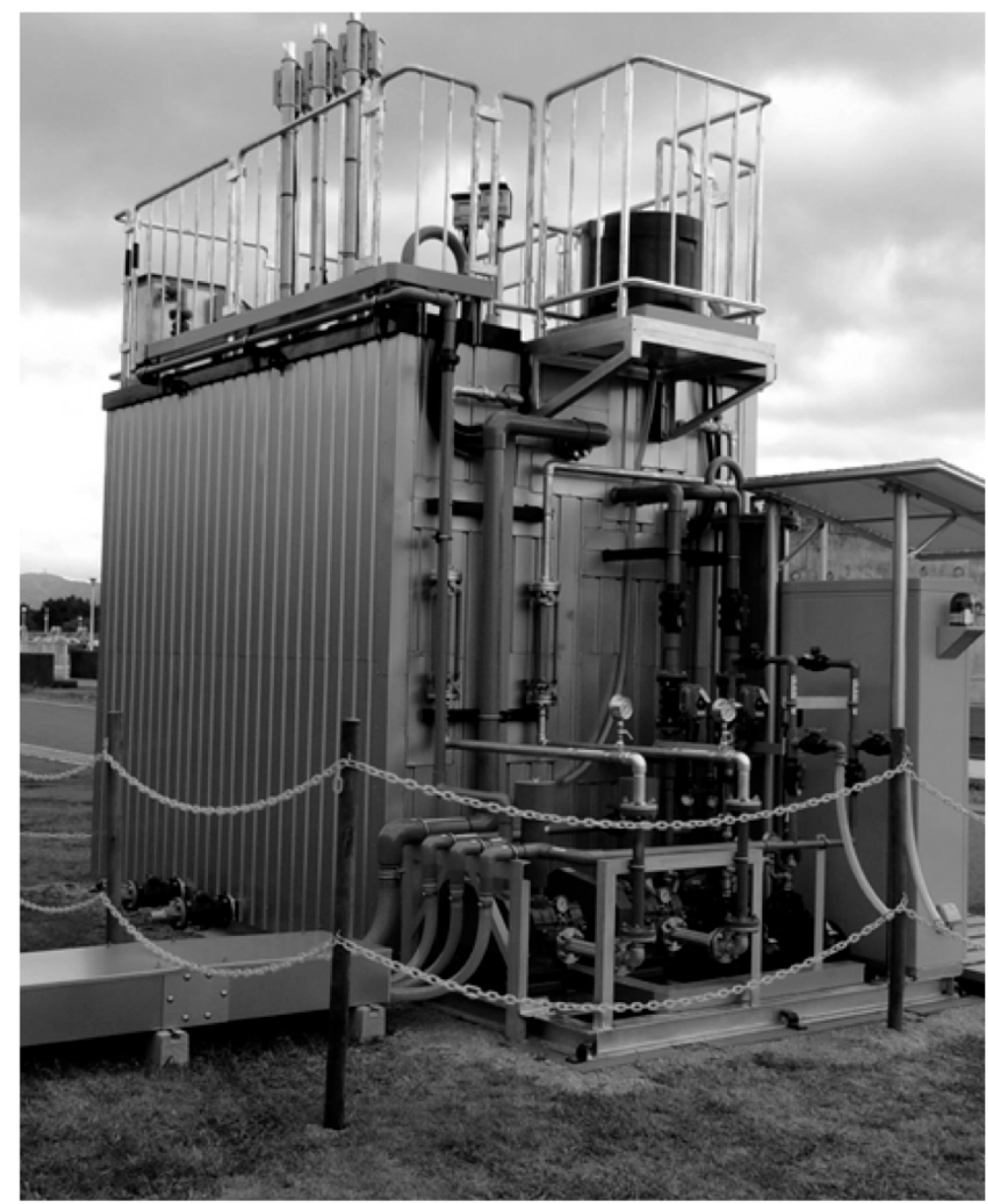

Figure 1: Pilot Plant of the membrane bioreactor.

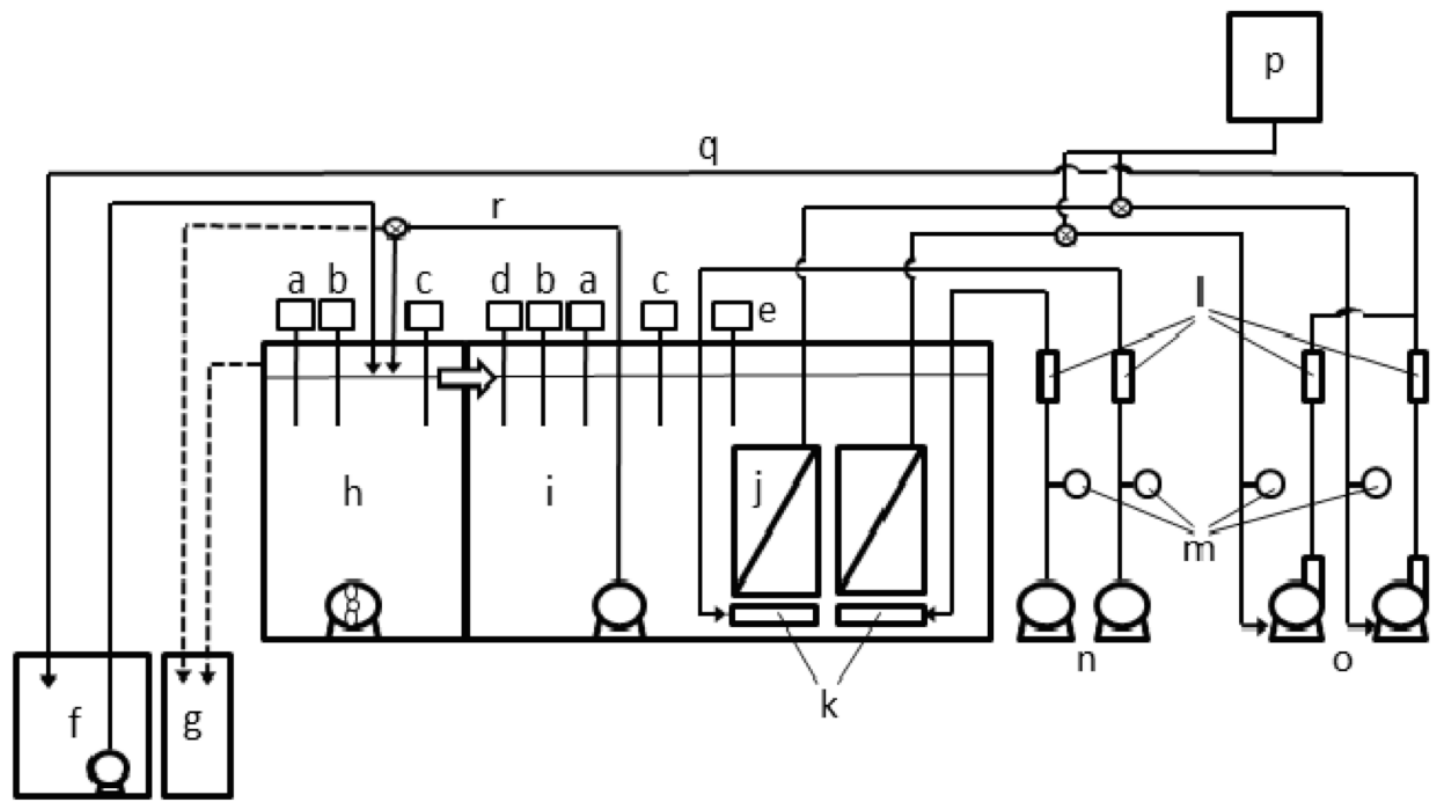

Figure 2: Block diagram of the membrane bioreactor pilot plant.

(a) $\mathrm{pH}$ indicator, (b) Oxidation-reduction (OR) potential indicator, (c) Liquid level indicator, (d) Thermometer, (e) Dissolved oxygen (DO) indicator, (f) Initial sedimentation tank, (g) Biological treatment bath, (h) Anaerobic bath, (i) Aerobic bath, (j) T2 membrane module, (k) Aeration tube, (l) Flow gauge, (m) Pressure gauge, (n) Aeration pomp, (o) Suction pump, (p) Tank for sodium hypochlorite solution, (q) Permeated water, (r) Line for returned activated sludge. 

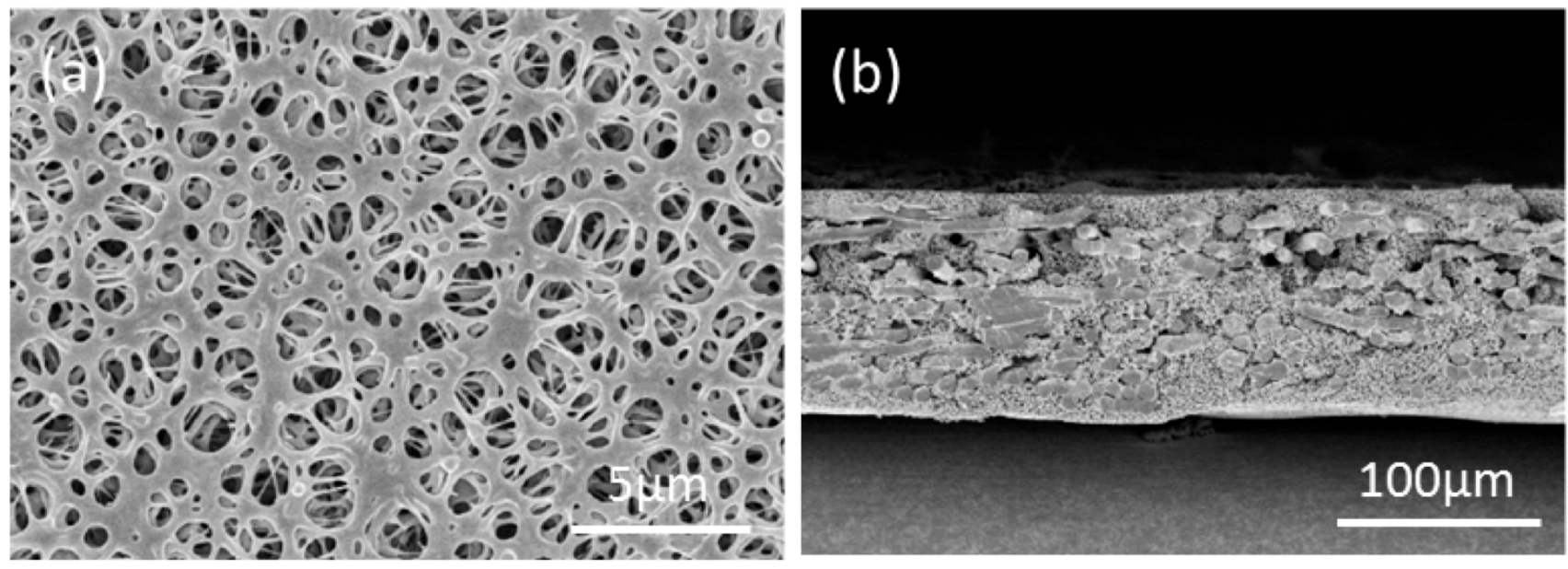

Figure 3: Scanning electron microscope (SEM) photographs for the membrane. (a) Surface, (b) Cross-section.

experiment has an average pore size of $0.3 \mu \mathrm{m}$ made from CPVC and polyester nonwoven [1]. The structure on the surface and the cross-section of membranes is shown in Figure 3 as a photograph of scanning electron micrograph. We can confirm that the surface of the membrane is covered with very thin fibrous structures of CPVC that form a network structure which plays a role of separation membrane having small pores of an average diameter of $0.3 \mu \mathrm{m}$. The size of the fibrils is in the range of $100-1000 \mathrm{~nm}$. The crosssection shows that the fibrous structure expands from the surface of the membrane towards the inner part but the density of the fibrils becomes less in the order from surface to core. There can be seen the cross-section of PET fibers that is part of a polyester nonwoven. A transmission electron micrograph for the cross-section of the membrane is shown in Figure 4. The specimen was stained with phosphotungstic acid to observe the structure of HPC from CPVC, because the stain can react with HPC selectively. We can confirm that fine HPC particles attached on the surface of the fibrils made from CPVC, which is one of structural characteristics of our membranes. It should be noted here that this structural characteristic of the membrane structure has not been reported yet in any scientific and engineering journals as far as the authors are concerned with and the novelty of this membranes lies here.

The CPVC flat sheet membrane was processed to form an element with a frame made from polyvinyl chloride (PVC). The frame has a relatively large rectangular dual-side thin shallow saucer (Figure 5). The CPVC flat sheet membrane is fixed on the four edges of the frame with the thermal-melt method. The water filtered with the membrane was suck up by a pump there. The size of the element is $1,000 \mathrm{~mm}$ length, $490 \mathrm{~mm}$ width, and $6 \mathrm{~mm}$ thickness.

The pilot plant has two individual lines and every line connects to a membrane unit (module) that contains 50 sheets of membrane elements each. They are installed in an aerobic bath. The membrane bioreactor consists of aerobic and anaerobic baths. To keep the concentration of mixed liquor suspended solids (MLSS) in activated sludge as a suitable level, the residual sludge in the bioreactor was removed periodically. A washing system with a chlorine solution
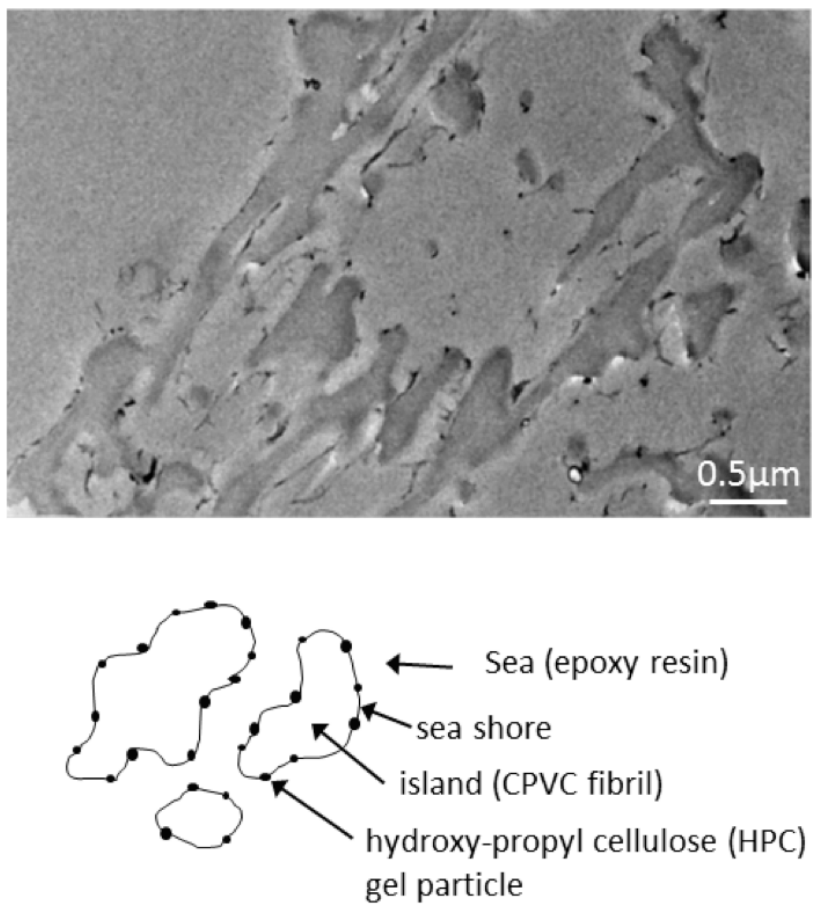

Figure 4: Transmission electron microscope (TEM) image for the cross-section of the membrane. 
was also equipped and the content of a sodium hypochlorite solution should be adjusted to its effective chlorine concentration of $0.6 \%$.

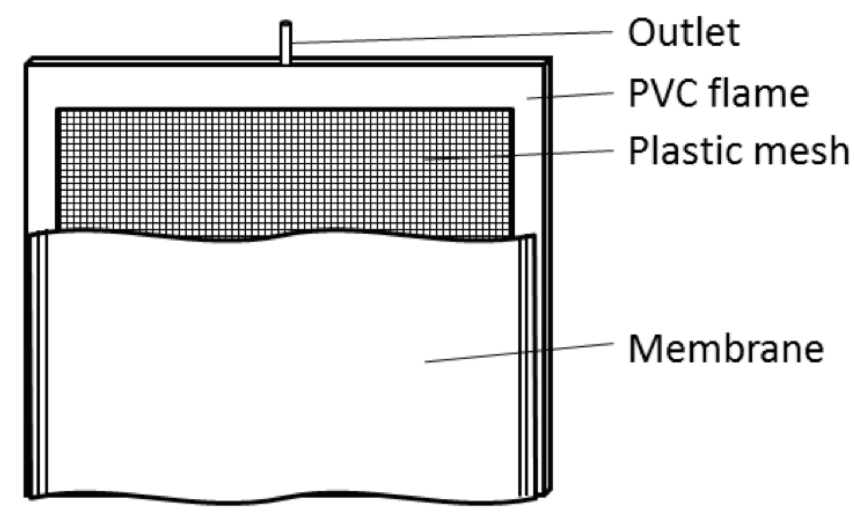

Figure 5: Schematic diagram of the membrane element.

The actual operation and measurement conditions of the pilot plant are as follows. The water was suck up with a suction pump through membranes and the pump was operated with the intermittent mode of continuous suction for 8 minutes and a halt for 2 minutes. This flux rate is widely used for operation in usual MBR systems, so we adopt the value for operation of our pilot plant test $[12,13]$. We keep constant flux and the change of TMP was measured with time. The surface of the membrane element was continuously flushed with water flow induced by bubbles made with an aeration system equipped on the bottom of the membrane unit to prevent fouling materials from sticking and depositing on it to form a cake layer in the end. This installation of the aeration system is important and operated continuously, because once fouling materials fills the pores of the membrane, the trans-membrane pressure rises abruptly. The intermittence of sucking water is expected to give some relaxation effect on generation of membrane fouling. It is also important to design the bubble size, because it is related to intensity and distribution of the water flow induced by the bubbles that wipes off the surface of the membrane element. The homogeneous flow without turbulence on the surface of the membrane will prevent foulants from depositing on the surface on the whole part of the surface of membranes without localization. Operation conditions are tabulated in Table $\mathbf{2}$. The temperature of the sewage in the bioreactor was not controlled but the actual measured temperature pointed at an stable value was in the range of $17-28{ }^{\circ} \mathrm{C}$ for over the whole one-year term of operation. We understand that the lower temperature of operation especially in a winter time may give a bad effect on the activity of microbes in the bioreactor and organic materials in the wastewater may not well metabolized. It produces an increase of extracellular polymeric substance (EPS). The EPS consists of polymeric materials such as polysaccharides and polypeptides that have a potential to deposit on the wall and close the pores of the membrane. It may be happened in the activated sludge and may become foulants that will deposit on the surface of the membrane and cause a blockade. Therefore, to keep the temperature of bioreactor over $15{ }^{\circ} \mathrm{C}$ as much as possible with less cost, the bioreactor was shrouded with a thermal insulator and the effect was confirmed to be well working. The resultant temperature was in the range at which microbes are active well to metabolize the organic

Table 2: Test Condition

\begin{tabular}{|c|c|c|}
\hline Parameter & Unit & Range \\
\hline \hline Flux & $\mathrm{m} / \mathrm{d}$ & 0.43 \\
\hline Intermittent mode & $\mathrm{LMH}$ & $8 \mathrm{~min}$ On, $2 \mathrm{~min}$ Off \\
\hline HRT & $\mathrm{h}$ & 9.1 \\
\hline Aeration flow rate & $\mathrm{L} / \mathrm{min}^{\prime} / \mathrm{unit}$ & $200-320$ \\
\hline Circulation ratio & $\mathrm{Nm}^{3} / \mathrm{m}^{2} \mathrm{~h}$ & $0.28-0.45$ \\
\hline Temperature & & $2-3$ \\
\hline pH & ${ }^{\circ} \mathrm{C}$ & $17-28$ \\
\hline DO & & $6.4-7.1$ \\
\hline MLSS & $\mathrm{mg} / \mathrm{L}$ & $0.4-7.1$ \\
\hline SRT & $\mathrm{mg} / \mathrm{L}$ & $8,300-18,000$ \\
\hline
\end{tabular}




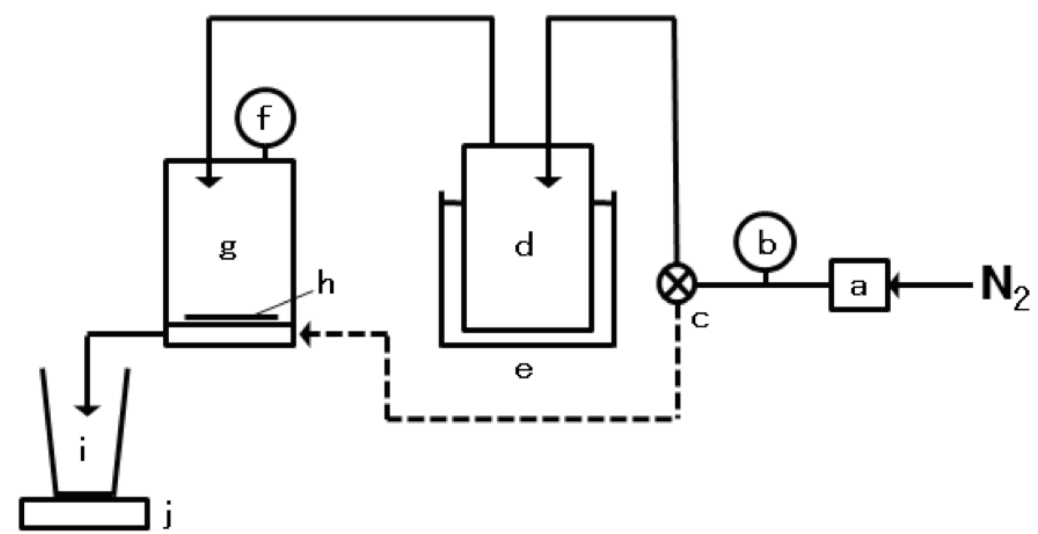

Figure 6: Schematic diagram for the evaluation apparatus to measure Flux and the bubble point.

(a) Pressure reducer, (b) Pressure gauge, (c) Switching valve, (d) Water supply tank, (e) Thermostatic bath, (f) Pressure gauge, (g) Evaluation vessel, (h) sample membrane, (i) Measuring cup, (j) Electronic balance.

materials in the sludge. The range of $\mathrm{pH}$ is $6.4-7.1$, the DO is $0.4-7.1 \mathrm{mg} / \mathrm{L}$ and the MLSS is 8,300 $18,000 \mathrm{mg} / \mathrm{L}$ during the one-year operation.

\section{Flux and the Bubble Point}

Flux and the bubble point of membranes are measured using an apparatus, the schematic diagram of which is depicted in Figure 6. A circle shape sheet membrane in a diameter of $90 \mathrm{~mm}$ which was cut from an original membrane sheet roll and was set on the bottom of the vessel in the apparatus, and then water purified with a reverse-osmosis (RO) membrane was poured into the vessel and sealed. Pressurized nitrogen gas was plunged into inside the vessel to push water at a pressure of $50 \mathrm{kPa}$ to get filtered water through the CPVC membrane for measurement of water flux. After passing the time of 30 seconds of opening the pressure valve, the sampling of water was started and the outflow water from the inside through the membrane was measured on a balance. When the sample membrane was hard to be wetted the lead time before measurement was extended until the outflow of water became stable. The water flux is estimated with the following equation.

\section{Pure water flux $\left[\mathrm{L} / \mathrm{m}^{2} / \mathrm{hr} / \mathrm{kPa}\right]=Q / A / P$}

where, $Q[L / h r]$ is the amount of outflow water through the membrane during the time of one hour, $A$ is the effective area of the membrane to filter water in the apparatus, and $P$ is the value of the applied pressure.

The bubble point is measured with the same apparatus. The procedure to set membrane and water is same but the setting is different. The applied pressure was increased from $0 \mathrm{MPa}$ at a rate of 0.02
$\mathrm{MPa} / \mathrm{min}$ from the opposite side of the water through the membrane. It is noted that the vessel should be designed to use a material having transparency to look into the surface of the membrane through the wall of the vessel. In the course of pressurizing water if we looked at the surface of the membrane on the bottom of the vessel we observed some hemispheres of air bubbles starting to form and they became a full sphere of bubbles as the pressure increased. Soon, they gave up sticking to the surface of the membrane and floated up when the pressure increased beyond a certain point. Here the bubble point is defined as the value of a minimum pressure at which the air bubbles are continuously generating and popping up from the surface of the membrane in the water of the vessel.

\section{Membrane Structural Analyses}

\section{SEM}

A scanning electron microscope (SEM) image was taken with an electron microscope. Carbon was coated after the sample membrane was set on a sample holder before observation. A S-4500 scanning electron microscope of Hitachi was used and an image as a magnifying ratio of 5,000 was taken for both the surface and the cross-section of membranes. To observe the cross-section the membrane was cut with a razor blade in liquid nitrogen. Then the sample was set on the sample holder and the plane of the crosssection was set to be faced up for observation.

\section{EDX}

A spectrum of energy dispersive X-ray spectroscopy (EDX) was obtained with an EMAX Energy spectrometer of Horiba attached to the same S-4500 SEM microscope in SEM observation. 


\section{TEM}

A commercially available epoxy resin (a mixture of Nakarai Ruabekku 812, Epicoat 812 and Ruabekku DMP-30) was adopted to embed a sample membrane before microtoming and the sample embedded in the epoxy resin was kept in an oven at $60{ }^{\circ} \mathrm{C}$ for 24 hours for curing. Then the membrane in the resin block was microtomed with a microtome (Reichert-Nissei Ultracut $\mathrm{N})$ and a diamond knife (Sumitomo Sumiknife). All the sections microtomed were collected on a carboncoated thin film. Then it was put on a sample holder for TEM (transmission electron microscope) observation. An electron microscope (JEOL JEM-2100) was operated at an accelerated voltage of $200 \mathrm{kV}$. The images were recorded on a CCD camera (Gatan Orius SC 1000).

\section{Infrared Spectrometry}

An infrared (IR) spectrum on the attenuated total reflection (ATR) mode was obtained with a Cary 670 FTIR spectrometer of Agilent Technologies equipped with a Thunder Dome ATR attachment of Spectratech.

\section{RESULTS AND DISCUSSION}

\section{The Change of Trans-Membrane Pressure (TMP) for One-Year Operation}

\section{The Initial Operation and its Observation in RUN 1}

The pilot-plant field test was performed for one year. In Figure 7 the TMP, the amount of aeration and the temperature are plotted against days. The terms of operation are tabulated in Table 3 . In the beginning (RUN1), the water was sucked up with a rate of $20-25$ $\mathrm{m}^{3} / \mathrm{D}$ from the bioreactor through membranes with an aeration rate of $200-250 \mathrm{~L} / \mathrm{min} / \mathrm{unit}(0.28-0.35$ $\mathrm{Nm}^{3} / \mathrm{m}^{2} \mathrm{~h}$ ). Please note that this amount of aeration is reduced to the level of less than $50 \%$ of the normal operation conditions many MBR manufacturers recommend or they are shown in the literature $[12,13]$. After one month operation the value of transmembrane pressure became much and they reached $30 \mathrm{kPa}$. We washed the membrane with a chlorine solution that was made from sodium hypochlorite and water as effective chlorine concentration of $0.6 \%$. The washing solution was poured into the inside of membrane elements from the outlet through pipelines from the upper part of the bioreactor with gravitation. The amount of washing solution is $100 \mathrm{~L}$, which can fill all the empty space inside the membrane elements according to the calculation based on its design. After leaving the time of 30 minutes the washing solution was discharged from the inside of the membrane element and the filtration of clear water was restarted. The pressure went down to 7 9 kPa after washing.

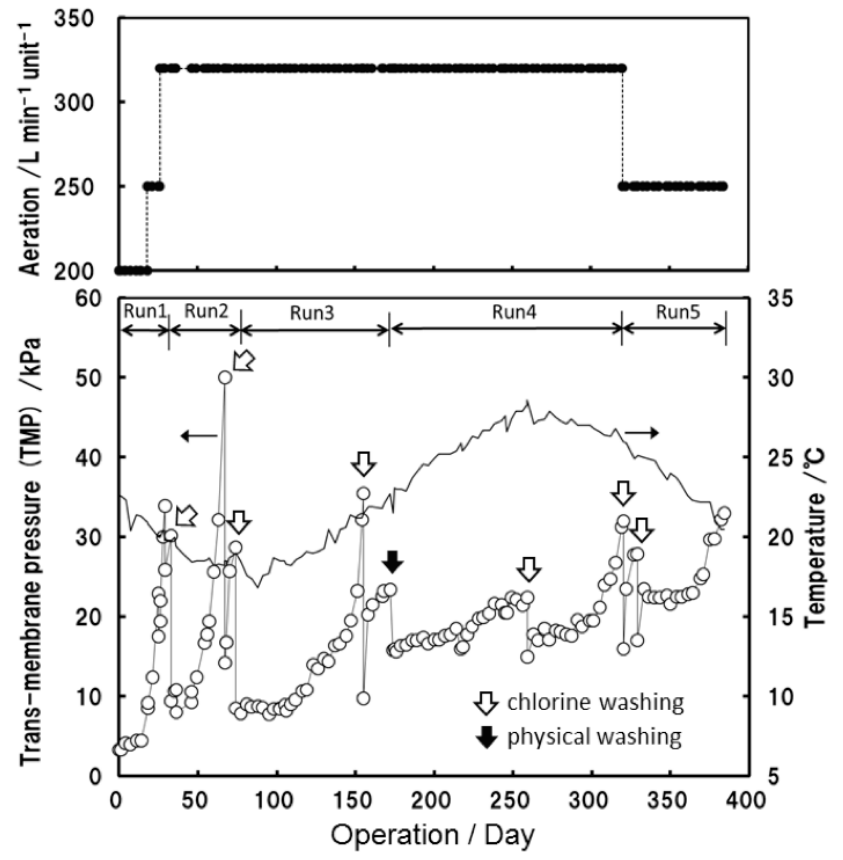

Figure 7: Change of trans-membrane pressure (TMP), temperature and aeration with time.

\section{The following Observation in RUN 2 after Chemical Washing}

After 30 days passing from the day of the washing with a chlorine solution the pressure rose again from 9 $\mathrm{kPa}$ to $50 \mathrm{kPa}$. In RUN2 the aeration rate was raised to $320 \mathrm{~L} / \mathrm{min} /$ unit $\left(0.45 \mathrm{Nm}^{3} / \mathrm{m}^{2} \mathrm{~h}\right)$, because we saw the gradual rise of TMP in the RUN1. There was the necessity to put relatively high efficient force of surface cleaning with much amount of water flow induced by bubbles and we expected a longer term operation with low TMP. Irrespective of changing operation conditions of the aeration rate we met again the pressure rise of TMP after just 30 days from the start in the last part of RUN2. The developed membrane was fouled again as almost the same duration time of RUN 1. The duration time of 30 - 40 days before the rise of TMP up to 40 $50 \mathrm{kPa}$ was shorter than that of expectation when we designed the pilot plant. We also discuss the means how the duration time is made longer and met our expectation with low TMP. There is a doubt that the structure of the developed membrane element may have some shortcomings in design and all the foulants that close the pores of the membranes cannot be removed completely with a chlorine washing process. It is true as an experimental fact that the value of TMP 
Table 3: Change of Trans-Membrane Pressure (TMP) for One Year

\begin{tabular}{|c|c|c|c|}
\hline Term & Days & Throughput of aeration air L/min/unit & Temperature ${ }^{\circ} \mathrm{C}$ \\
\hline \hline RUN 1 & $1-30$ & $200-250$ & $20-22$ \\
\hline RUN 2 & $30-67$ & 320 & $19-20$ \\
\hline RUN 3 & $68-172$ & 320 & $18-23$ \\
\hline \multicolumn{7}{|c|}{ Physical Washing with a sponge } \\
\hline RUN 4 & $173-320$ & 320 & $23-29$ \\
\hline RUN 5 & $321-384$ & 250 & $20-28$ \\
\hline
\end{tabular}

decreases after washing with a chlorine solution, but we are afraid of inhomogeneity in that some part on the membrane is cleaned well and foulants that close the pores is washed out but some is still closed without removal of foulants. The reason why such malfunction in a chlorine solution process occurs is that the resistance for the liquid flowing inside the membrane elements may be high and all the fouled part isn't washed well because the chlorine liquid can't reach the whole area of the membrane due to the bad channeling flow of the washing solution. Improvement of element structure is required to promote the flow of the washing solution inside the element to make membrane washed well. This will be also discussed in a future article to design a new structure for membrane element and its advantages. To overcome this disadvantage with the present element structure we adopted a twice longer time and a twice amount of chlorine liquid for membrane washing. This revised chlorine washing procedure was performed in the end of RUN 2, and then the test was resumed.

\section{The Behavior in RUN 3 after the Procedure of Chemical Washing is Changed}

This next term (RUN 3) started after chemical washing with the revised condition in RUN2. The increase of TMP is observed to be less than that of the previous periods (RUN 1 and RUN 2) and the developed membrane element can be operated stably for 80days before we confirm the raise of TMP. It is considered that the increase of washing liquid in quantity and the time to wash fouled membranes with the present element structure is effective. The most fouled part that is still fouled after the previous procedure of washing on the membrane will be cleaned. However, it should be noted here that the chlorine washing was made in the last part of RUN 3 (on the 155th day) but the TMP decreased $35 \mathrm{kPa}$ to $10 \mathrm{kPa}$. Here, the time and the volume of washing solution was adopted the same condition in RUN 2. There seems some foulants still deposited after chlorine washing and the pressure didn't recover the zero pressure value. It is important to see if what are the reasons to give a non-zero TMP after chlorine washing.

The test (RUN 3) was once finished on the 172th day and all membrane elements were removed from the bioreactor. The surface of the membrane was wiped off physically with a sponge to remove cake layers made from foulants on it. The photographs in Figure 8 show the surface of the membrane element before and after the removal of cake layers when the elements are removed from the bioreactor and pictured on the day. It is confirmed with eyes that all part on the surface of the membrane after physical washing with a sponge seems to be not covered with cake layers. If we look into the surface of the membrane after physical washing, the surface is confirmed to consist of gray and white color parts and some stream-like patterns appear as bands of the white color, which may correspond to the flow of water in a bioreactor. The gray part is the place in which fouling material will be still adhered to the membrane. The flow of water speed is not homogeneous over the surface of the membrane and insufficient to prevent foulants from depositing on the membrane. The relationship between this inhomogeneity of water flow and the adhesion of foulants on the surface will be discussed again in a later section.

All membrane elements were re-installed after physical washing in a bioreactor and the test was resumed on the same day. It is noteworthy to point out that the TMP value after physical surface washing with a sponge shows not zero value and it becomes larger than the initial value of its virgin (initial) state by $16 \mathrm{kPa}$. This is because the irreversible component of fouling that cannot be removed with both chlorine and physical washing and may still deposit on the pores of the membranes, which may cause an increase of resistance for flowing water through the pores. 

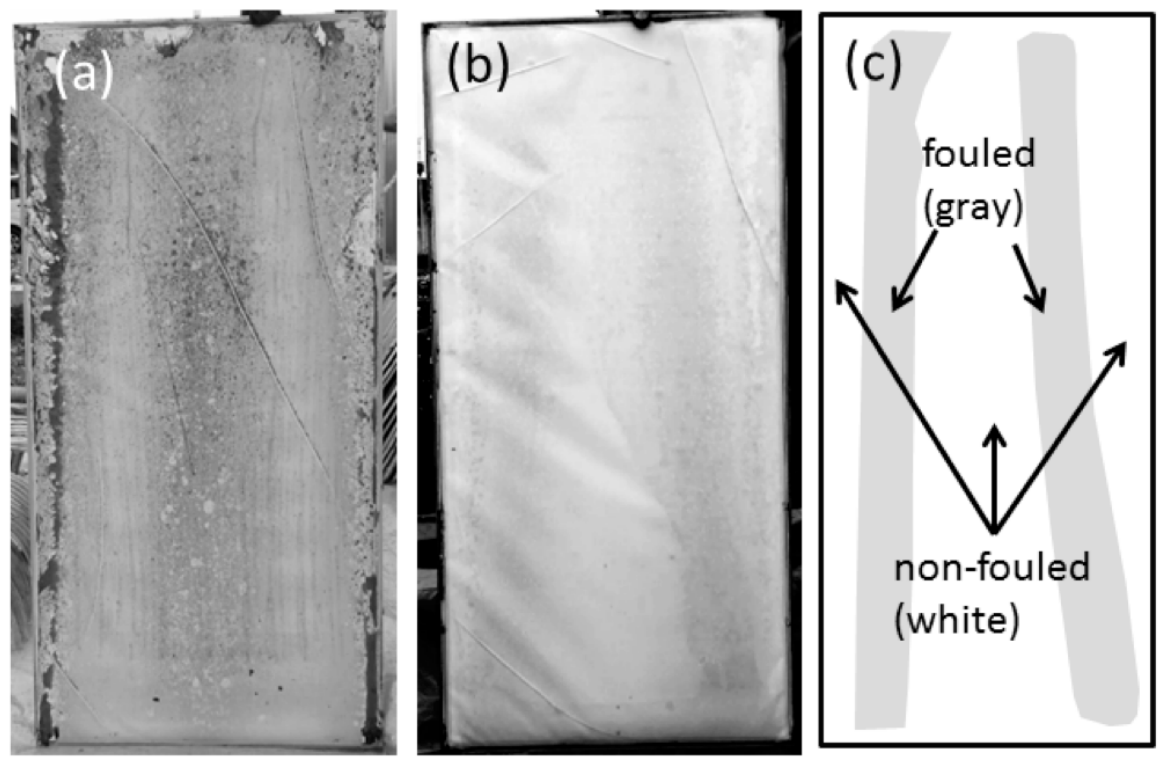

Figure 8: Membrane element after half-year use in the bioreactor.

(a) Just removed from the bioreactor, (b) After wiping off physically with a sponge, (c) Schematic diagram to show the fouled and un-fouled parts for the picture in (b).

\section{After Physical Washing in RUN 4}

After the restart (RUN 4) the membrane shows a stable and almost constant TMP for four months. The pressure gradually increases from $16 \mathrm{kPa}$ to $22 \mathrm{kPa}$. A washing with a chlorine solution (same condition in RUN 2) was carried out on the 259th day but TMP didn't change much before and after the washing. We think that the judgment to wash membranes on the day is a miss operation and the chlorine washing is not necessarily required because no change of TMP was observed as a result. It is considered that the removal of cake layers by physically wiping with a sponge on the 172th day contributes to the stabilization of operation and keeping the surface of membranes clean from foulants, which prevents the cake layer from depositing on the surface. There is a possibility that some part on the surface seems to be covered with cake layers after a chlorine washing process was performed, which might give a shorter time when the rise of TMP comes before physical washing in the term of RUN 1 - 3. The value of TMP had been kept low since the physical washing in the end of RUN 3 was carried out. In the last period of Run 4 the pressure rised up to $32 \mathrm{kPa}$, so the chlorine washing (same condition in RUN 2) was made on the 320th day. The value of TMP came down to $16 \mathrm{kPa}$. It should be noted that the membrane achieves a stable operation for 4 months to keep the low TMP value with a relatively low amount of aeration $(320 \mathrm{~L} / \mathrm{min} / \mathrm{unit})$ under the condition in which even the inner element structure is needed to be re-designed.

\section{More Reduction of Aeration Rate in RUN 5}

In the last run from (RUN 5) the aeration rate was decreased to $250 \mathrm{~L} / \mathrm{min} / \mathrm{unit}\left(0.35 \mathrm{Nm}^{3} / \mathrm{m}^{2} \mathrm{~h}\right)$ to see the effect of saving electricity more. It should be noted that TMP didn't increase so much and we could achieve a stable operation for two months. All membranes were removed from the bioreactor to analyze the state of fouling with physical and chemical methods after the period was finished. New membrane elements with an improved inner structure to prevent the channeling flow inside from occurring were installed and the measurement was started.

\section{Quality of Treated Water}

The results of analyzing water quality before and after treatment with membranes over one year are shown in Figure 9. The parameters of BOD, COD, SS, E. coli, total nitrogen (T-N), and total phosphorus (T-P) is analyzed. It is seen that all parameters are much reduced after treatment in a bioreactor and the filtered water shows less contaminated. When we look into the curve of total nitrogen for treated water in the figure, it changes from increase to decrease on the 215th day. This change coincides with the time when the rate of withdrawing residual sludge from the bioreactor is changed from $160 \mathrm{~L} / \mathrm{d}$ to $240 \mathrm{~L} / \mathrm{d}$. The components containing nitrogen will be removed with a removal of sludge. The curve abruptly goes up on the 308th day. This change is in agreement with the operation change of aeration rate from $320 \mathrm{~L} / \mathrm{min}$ to $250 \mathrm{~L} / \mathrm{min}$. Because 

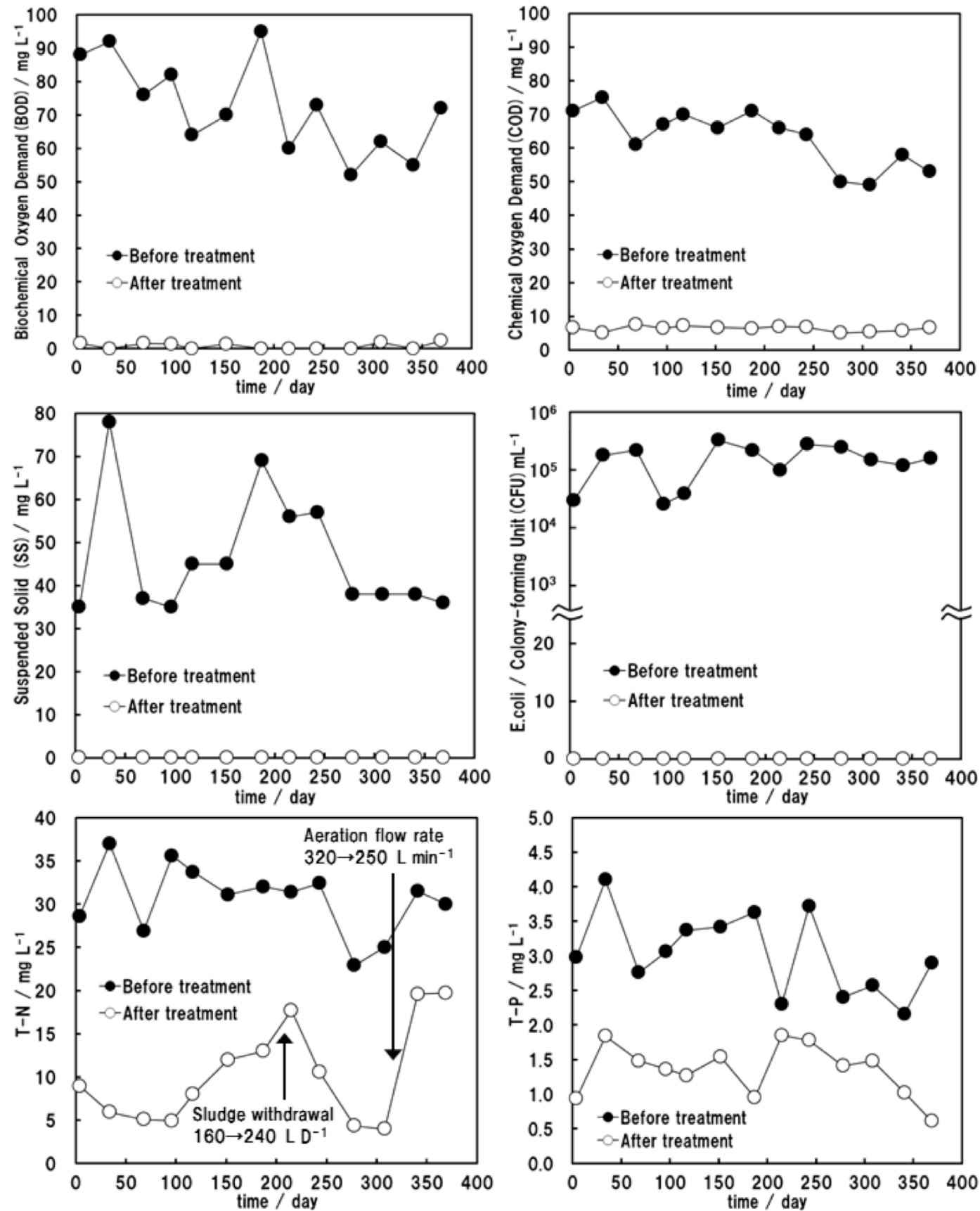

Figure 9: Results of water quality before and after treatment over one year.

the aeration rate is reduced, the total concentration of oxygen in water in the bioreactor will be reduced and the reaction of nitrification may receive a bad effect due to lack of oxygen. This is a speculation to explain why the concentration of total nitrogen in treated water goes up.

\section{Physical and Chemical Analyses of Membrane after One Year Use}

The membranes removed after one-year test from the bioreactor were analyzed physically and chemically. Figure 10 shows the photographs of the membrane elements after use. The surface of the membrane was wiped off physically with a sponge to remove cake layers of the activated sludge attached on the element after removal from the bioreactor. It is interesting to point out that the surface is partly covered with some gray color materials but some part shows a white color as a fresh CPVC membrane does, indicating that the surface of the membrane is well made hydrophilic and the foulants together with the cake layer deposited on the surface can be easily 


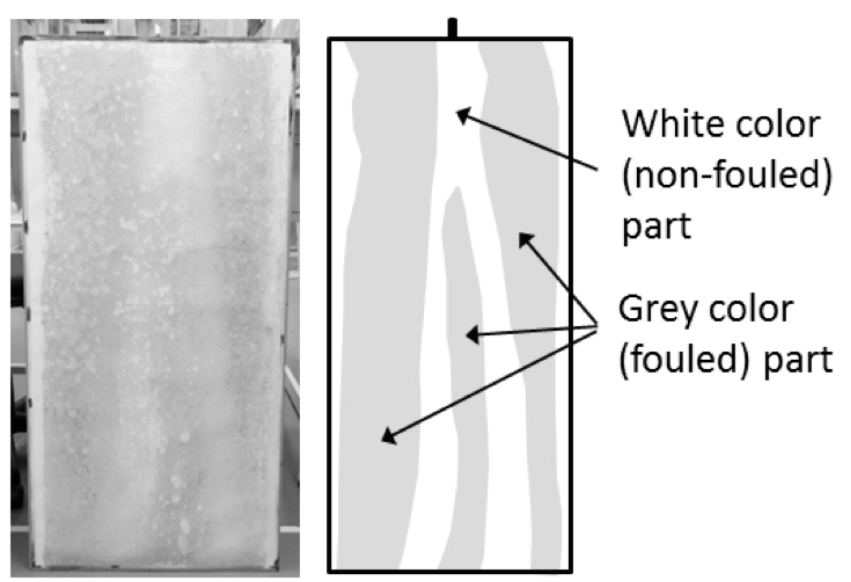

Figure 10: Membrane element after one-year use in a bioreactor after wiping off physically with a sponge.

detached with physical washing. The gray color part seems to be partly closed with foulants. The white part is considered in which the fresh facet of the original membrane appears after detachment of cake layers. This detachment of the foulants can be realized by the effect of the surface structure made from HPC. A flux test with pure water was performed and the result is tabulated in Table 4 . It should be noted that the membrane developed by us after one year operation can be wetted in no time with water without pretreatment when it is immersed in water on the apparatus in Figure 6, indicating that the hydrophilicity of the membrane is maintained after one-year use. We can see that the white part shows almost same values of flux and the bubble point with a fresh membrane, while the flux for the gray color part is about the half of the fresh membrane but the bubble point keeps the same level. This fact tells that the white part keeps almost the same properties of a fresh membrane. The white color part on the surface of membranes may show the result of the flow patterns of water that washes the surface of the membrane induced by aeration bubbles. It is probable that two streams starts from the bottom of the membrane unit and they come closer each other in the middle height of the membrane element, then they are merging the one stream on the top. There may be a chance to achieve a longer operation time without rise of TMP if this white (nonfouled) area is made wider adjusting water flow on the surface of membrane induced by aeration in a bioreactor.

\section{Observing and Analyzing the Foulants on the Membrane}

The observation and comparison of membrane surface before/after physical washing with a sponge is performed. The part the cake layer was well removed and showed a white color was selected for a specimen after washing. Figure 11 shows SEM micrographs with a magnifying ratio of 5,000 . It is seen that the foulants are deposited and form a thin layer on the surface of the membrane before physical washing. The layer covers all the surface of the membrane and one cannot see any network structures of the original membrane surface. If we look at the picture of the cross-section, the layer of foulants is confirmed to be deposited on the very top surface of the membrane and doesn't penetrate into the inside. On the contrary, the picture after the surface washing shows that there is a network structure with an open-pore structure as is shown in the fresh membrane. This means that the layer of foulants that covers the surface of the membrane before washing is almost removed. This easiness of removal is also confirmed on the picture of the cross-section in

Table 4: Flux and the Bubble Point for Developed Membrane after One-Year Use in a Bioreactor with Washing Physically with a Sponge

\begin{tabular}{|c|c|c|c|}
\hline Position measured & color & Flux L/m $/ \mathbf{h r} / \mathbf{k P a}$ & Bubble Point MPa \\
\hline \hline initial (average) & white & 162 & 0.155 \\
\hline Left top & gray & 97 & 0.175 \\
\hline middle & gray & 73 & 0.168 \\
\hline bottom & white & 121 & 0.175 \\
\hline Center top & white & 163 & 0.175 \\
\hline middle & white & 150 & 0.175 \\
\hline bottom & white & 144 & 0.175 \\
\hline Right top & gray & 98 & 0.175 \\
\hline middle & gray & 94 & 0.170 \\
\hline bottom & white & 164 & 0.170 \\
\hline
\end{tabular}




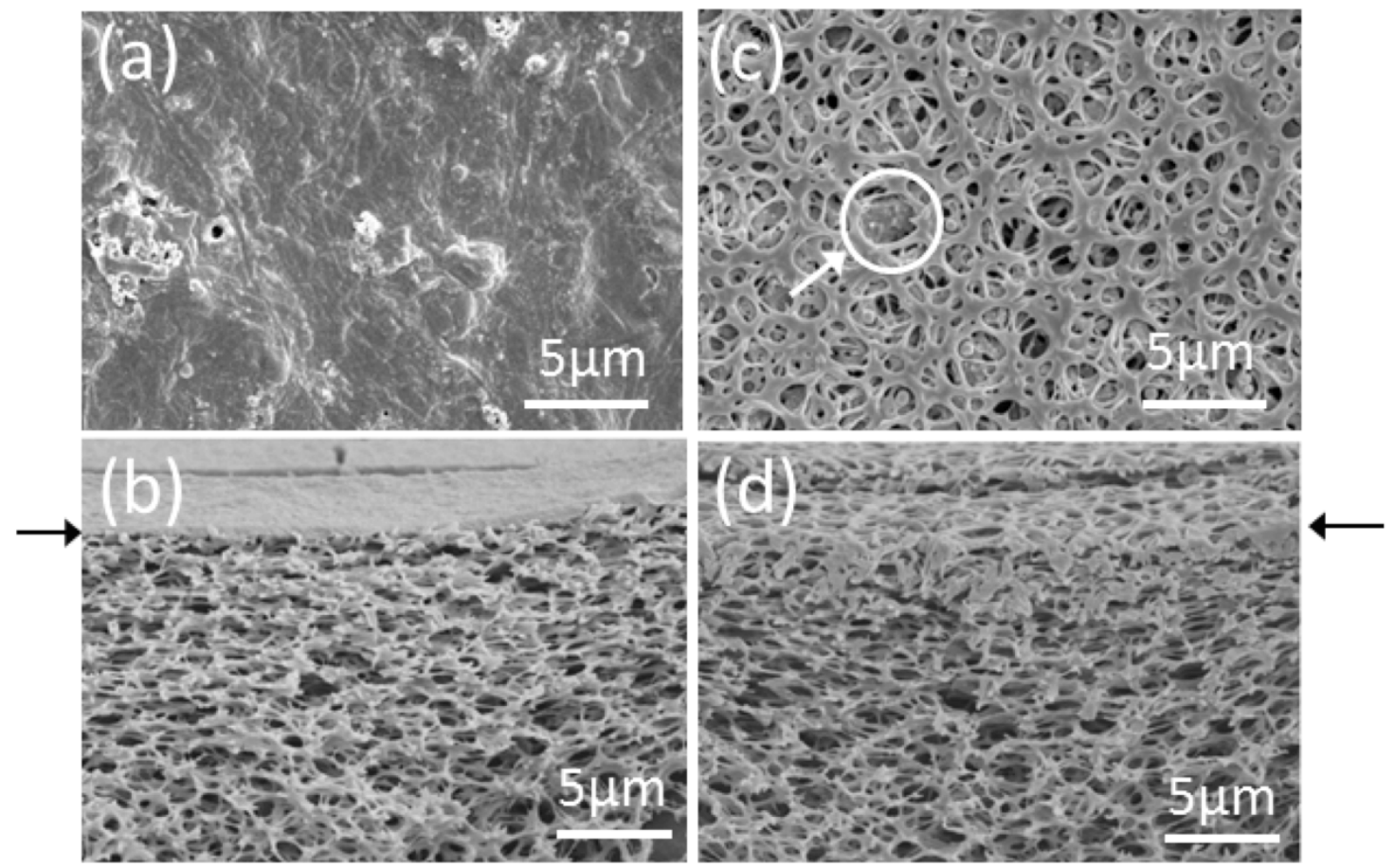

Figure 11: Scanning electron microscope (SEM) photographs for the membrane after one-year use in a bioreactor. (a) Surface (no-washing, x5,000). (b) Cross-section (no-washing, x5,000). The arrow points to the top of the cross section (c) Surface (after physical washing, $\times 5,000)$. A tiny trace of residual foulants is pointed with the white circle. (d) Cross-section (after physical washing, $x 5,000)$.

which no layer is confirmed on the very top of the membrane surface. However somewhere there seems to remain a tiny trace of foulants on the top just underneath the surface, one of the examples is pointed to with a white solid circle in the picture. With physical wiping the foulants on the surface is almost removed but there remains a tiny trace of foulants just beneath the surface.

To analyze chemically the layer of deposited foulants before washing and the remaining tiny trace of foulants after washing, infrared spectroscopy with ATR mode and EDX were applied. The results are shown in Figure 12 (IR) and Figure 13 (EDX). The IR spectrum before washing shows that the surface is covered with polypeptides and polysaccharides. There are almost no peaks detected due to CPVC, indicating that almost all surface is covered with cake layers. The EDX spectrum before washing indicates that the layer contains much inorganic materials made from calcium, phosphorous, potassium and magnesium. But the situation for the spectrum after washing is different and there is almost no inorganic component, indicating that most foulants made from inorganic materials is removed with physical washing. In the IR spectrum after washing the pattern is almost close to that of the fresh membrane. Most peaks are assigned to CPVC but some tiny traces of polypeptides or polysaccharides are confirmed. The
EDX spectrum also gives a same tendency and most peaks are assigned to chlorine that is due to CPVC and a very small amount of iron is confirmed. These facts suggest that the foulants deposited on the surface is cleaned through a physical washing process with a sponge but some tiny debris resides just beneath the surface and cannot penetrate into the inner part, which are detected on the IR and EDX spectra. The SEM micrograph on the cross-section after washing also suggests that the foulants doesn't penetrate into the inner part of membranes in the depth direction.

\section{Comparison with a Commercial CPVC Membrane Made by another Company}

In fact we installed commercial CPVC flat sheet membrane elements made by another company in a bioreactor in parallel as compared with our developed membranes and monitored TMP values with time over one year. The conditions for operation are exactly same as that of our products. After the one year use, the element was also removed from the bioreactor and the cake layer on the surface of membranes was wiped off physically with a sponge. The closed part with fouling materials on the membrane of a gray color is also confirmed, the area of which is much wider than that of our developed membranes and foulants cover almost $100 \%$ surface of membranes. The membrane 

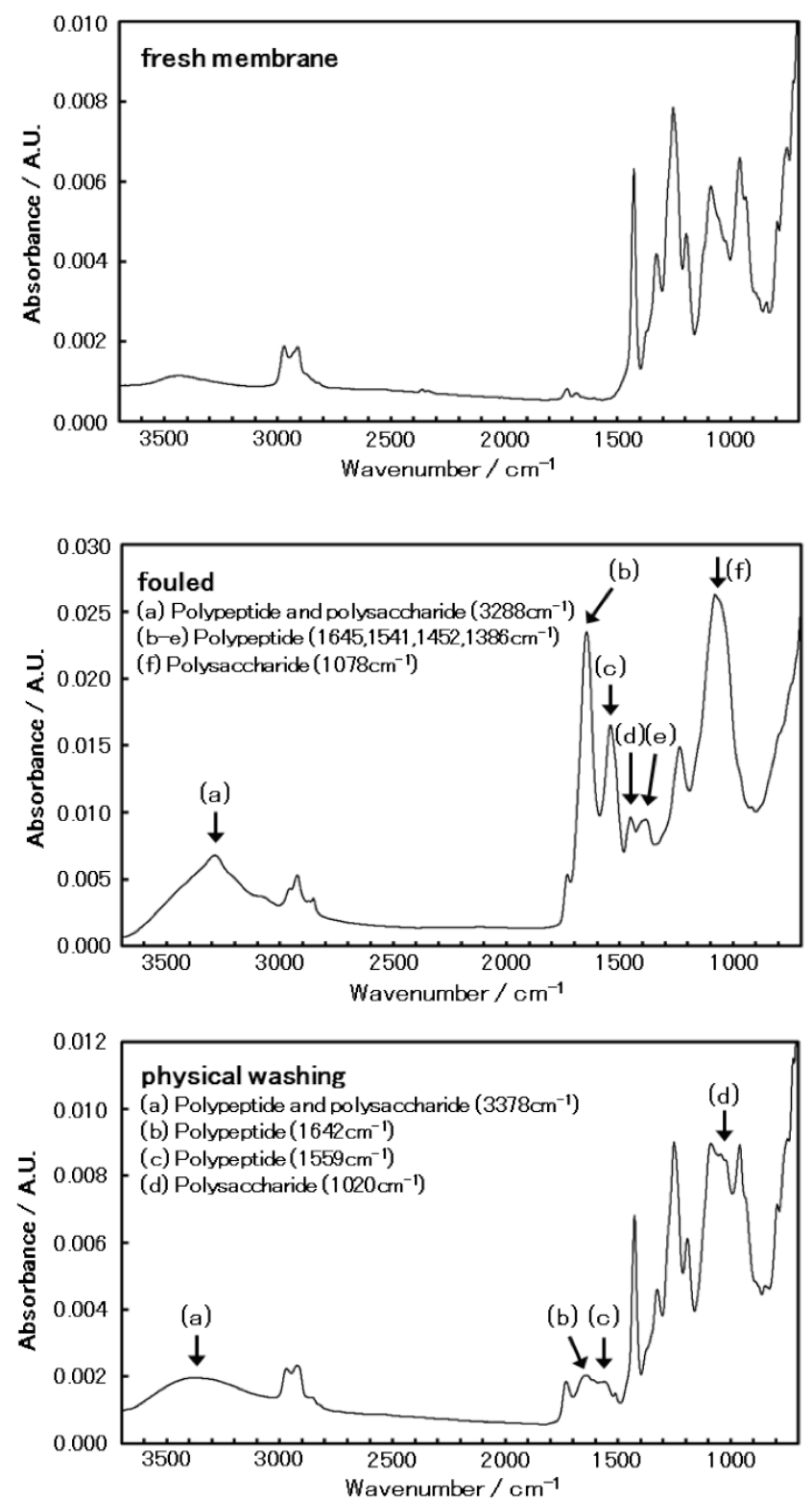

Figure 12: Infrared (IR) spectra for the surface of fresh, fouled and physically washed membranes.

was not wetted well in a short time with water in the apparatus to measure flux and the bubble point, indicating that it lost its hydrophilicity after one-year use in a bioreactor. The results of measuring flux and the bubble point on average for the whole area of the element is tabulated in Table $\mathbf{5}$ to compare the results between the developed and the commercial membranes. The developed membrane shows much water flux with its high retention. It should be also noted that about $35 \%$ of the surface area of the membrane developed by us shows white (without foulants) for the developed membrane. Even the fouled part showing a gray color after physical washing was wetted in no time for the developed membranes. These facts suggest
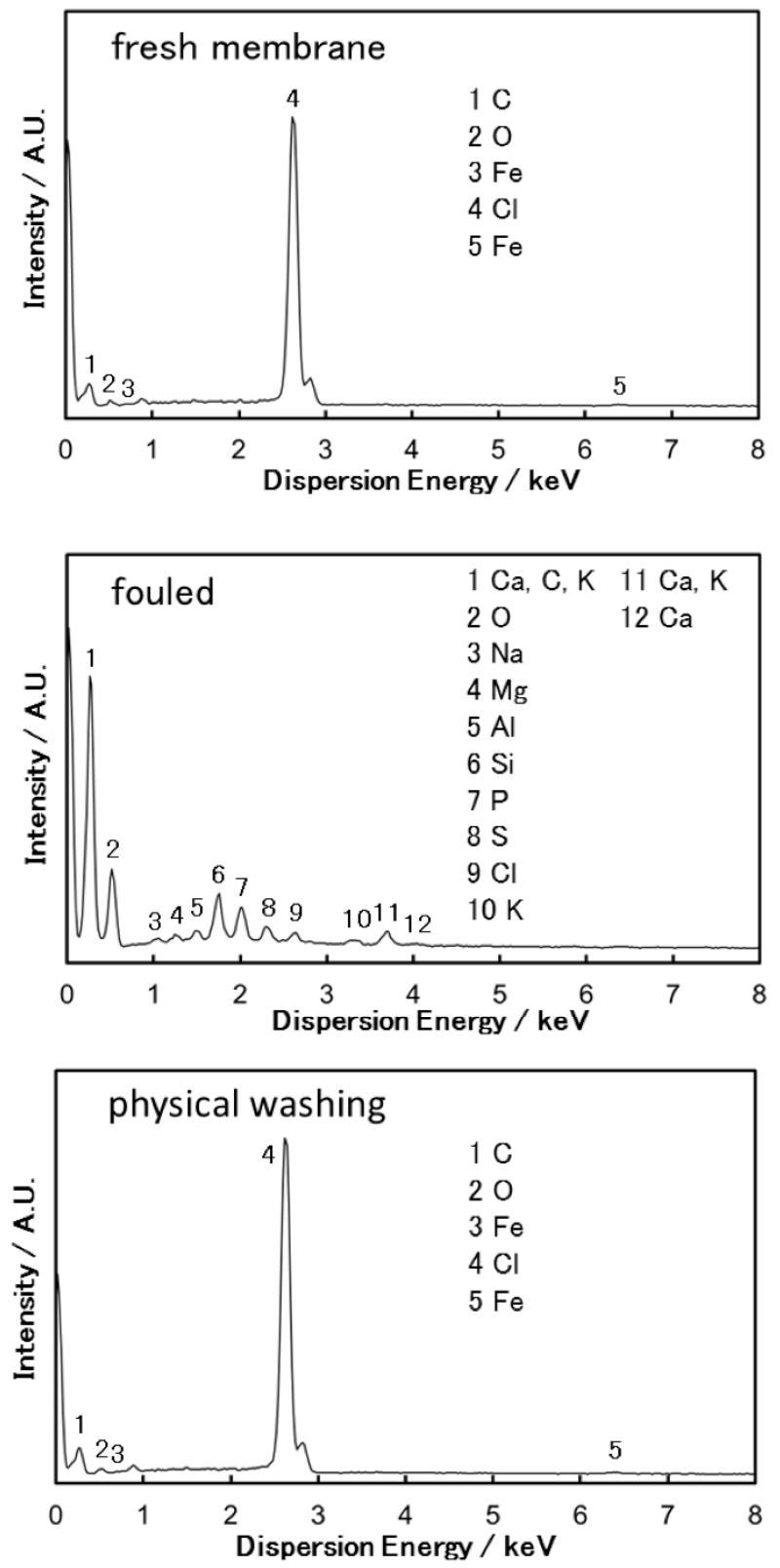

Figure 13: Energy dispersive X-ray (EDX) spectra for the membrane surface.

that our developed membranes have a tendency to keep lower fouling properties together with much more hydrophilicity than that of the commercial membrane produced by another company examined.

\section{Estimation for the Consumption of Electricity Especially for Low Aeration Air Operation}

Generally the MBR system is considered to be reliable because it produces high quality water to exclude possibilities of contamination with bacteria but the cost for electricity to drive the aeration system is a big issue. This time we performed a long time operation with less aeration air to estimate how much was the 
Table 5: Comparison in Flux and the Bubble Point between Developed and Commercial CPVC Membranes after OneYear Use in a Bioreactor. The Samples are Estimated after Physical Washing

\begin{tabular}{|c|c|c|c|}
\hline Sample & Flux L/m ${ }^{2} / \mathbf{h r} / \mathbf{k P a}$ & Flux Retention \% & Bubble Point MPa \\
\hline \hline Developed & 162 & & 0.155 \\
\hline Initial & 123 & 76 & 0.173 \\
\hline After use & 140 & 39 & 0.150 \\
\hline Commercial & 55 & 39 & 0.145 \\
\hline After use & \multicolumn{3}{|c|}{} \\
\hline
\end{tabular}

consumption of electricity. We monitored the consumption of electricity and the amount of produced water with time and calculated the electricity needed to produce a volume of $1 \mathrm{~m}^{3}$ water. In fact our system needs an amount of $2.0 \mathrm{kWh}$ electricity to produce $1 \mathrm{~m}^{3}$ water in total $\left(2.0 \mathrm{kWh} / \mathrm{m}^{3}\right)$ based on the collected data. However, if only the consumption for the aeration system is considered, the rate is estimated to be 0.8 $\mathrm{kWh} / \mathrm{m}^{3}$. In the literature the best figure for the total electricity consumption is reported to be $0.4 \mathrm{kWh} / \mathrm{m}^{3}$. As for the total consumption the rate $\left(2.0 \mathrm{kWh} / \mathrm{m}^{3}\right)$ for our system seems to be disadvantage if it is compared with the bench mark value of $0.4 \mathrm{kWh} / \mathrm{m}^{3}[14,15]$. Please note that the bench mark value was produced in a large facility to enjoy a scale merit equipped with a tall membrane module to utilize the water flow induced by air bubbles made with aeration apparatus. The large scale facility may make low the consumption of energy in incidental equipment like pumps, sensors and monitors as much as possible and the tall module also contributes to saving the energy consumption in aeration. On the contrary, our system is small $(0.7 \%$ of the bench mark system) in size and the height of the module is short (50\% of the bench mark system). If our system can enjoy the scale merit as the bench-mark test adopts a tall module, the electricity consumption should be reduced much close to the level of the bench mark with our developed membranes. As we mention in the previous part we continue to collect data in the pilot plant with improved membrane elements. They will show a stable operation with lower volume of aeration air and stand without membrane fouling with less chemical washing, which reduce the operation cost. The comparison of the total cost with the bench mark figure will be performed again in future.

\section{CONCLUSIONS}

A pilot-plant test for a membrane bioreactor was performed for one year with a newly-developed CPVC membrane in which its hydrophilicity was put with HPC.
It is proved that the novelty of the membrane structure lies in the configuration formed with CPVC fibrils and fine particles of HPC gel attached homogeneously on the surface of them. The pilot plant has been operated stably for one year with this novel membrane without much difficulty. The quality of the filtered water is proved to be good.

After one-year use the membrane tested was pulled out of the bioreactor and analyzed in detail with physical and chemical methods. The comparison between membranes developed by us and commercially produced by one of other manufacturers was made. The developed membrane shows less fouled than that of commercial ones. It should be noted that the cake layers made of foulants sticking on the surface of the membranes easily come off when they are washed physically with a sponge. The surface of the membrane after physical washing shows that there is almost no cake layer remaining on the surface. This nature will depend on the membrane having open-pore structure with much high porosity and homogeneouslydispersed small HPC gel particles attached on the surface of CPVC fibrils.

During this pilot-plant test our membranes developed show a stable operation and usefulness in an actual bioreactor. It is estimated that an amount of $2.0 \mathrm{kWh}$ for electricity is needed to filter $1 \mathrm{~m}^{3}$ of water through membranes and the figure is considered to be good as the system is considered to be categorized in a medium-size facility. The improvement of an element structure has been already performed and its estimation is ongoing in the following pilot-plant MBR test. The effect of saving energy with the new element will be revealed in the near future.

\section{ACKNOWLEDGEMENTS}

The authors would like to express their great gratitude to Shiga Prefectural Government for its comprehension and guidance to allow this pilot-plant 
test in Konan-Chubu Wastewater Treatment Plant. Thanks are also extended to the support and help of Public Interest Incorporated Foundation of Ohmi Environmental Conservation. Chemical and Physical analyses were made possible by the aid of the people in Analytical research center in Toyobo Co., Ltd., which should be especially acknowledged.

\section{REFERENCES}

[1] Higashi M, Morita J, Shimada T, Kitagawa T. Newlydeveloped Long-term-hydrophilic Flat-sheet Type Micro Filtration Membrane Made with Chlorinated Poly (Vinyl Chloride) (CPVC). in submission

[2] Werbowyj R, Gray D. Ordered Phase Formation in Concentrated Hydroxypropylcellulose Solutions. Macromolecules 1980; 13: 69-73. http://dx.doi.org/10.1021/ma60073a014

[3] Judd S. The MBR book, second edition. Elsevier 2011

[4] Nakahara T. MBR Technology, and Hollow Fiber Membrane Products. Membrane 2012; 37: 102-105. http://dx.doi.org/10.5360/membrane.37.102

[5] Xu Z, Qusay F. Polyethersulfone (PES) hollow fiber ultrafiltration membranes prepared by PES/non-slovent/NMP solution. J Membr Sci 2004; 233: 101-111. http://dx.doi.org/10.1016/j.memsci.2004.01.005

[6] Sik D, Kang J, Lee Y. The influence of membrane surface properties on fouling in a membrane bioreactor for wastewater treatment. Sep Sci Techno 2004; 39: 833-854.

[7] Kang J, Kim K, Lee Y. Preparation of PVP immobilized microporous chlorinated polyvinyl chloride membranes on fabric and their hydraulic permeation behavior. J Memb Sci 2003; 214: 311-321. http://dx.doi.org/10.1016/S0376-7388(02)00597-5

[8] Kang J, Kim K, Lee Y. Preparation of microporous chlorinated poly(vinyl chloride) membrane in fabric and the characterization of their pore sizes and pore-size distrubutions. J Appl Polym Sci 2002; 86: 1195-1202. http://dx.doi.org/10.1002/app.11063

[9] Kang J, Lee $\mathrm{S}$, Hu H, Shim J, Lee Y. Preparation of chlorinated poly(vinyl chloride)-g-poly(N-vinyl-2-pyrrolidinone) membranes and their water permeation properties. J Appl Poly Sci 2003; 88: 3188-3195. http://dx.doi.org/10.1002/app.12148

[10] Uesaka T. Membrane Separation: MBR. Kagaku Kogaku 2009; 73: 79-82.

[11] Witte P, Dijkstra P, Berg, J, Feijen, J. Phase separation processes in polymer solutions in relation to membrane formation. J Membr Sci 1996; 117: 1-31. http://dx.doi.org/10.1016/0376-7388(96)00088-9

[12] Guglielmi G, Chiarani D, Saroj D, Andreottola G. Impact of chemical cleaning and air-sparging on the critical and sustainable flux in a flat sheet membrane bioreactor for municipal wastewater treatment. Water Sci Techno 2008; 57: 1973-1879.

http://dx.doi.org/10.2166/wst.2008.126

[13] Nishimori K, Masutani H, Uejima T, Nagano M, Livingston D Izumi K, Technical innovation of KUBOTA submerged membrane unit. Proceed Wat Envir Feder 2005; 558-581.

[14] Nakagawa Y. Kubota MBR system for medium-size use. Industrial Machinery 2012; 5: 6-8.

[15] Krzeminski P, Graaf H, Lier, J. Specific energy consumption of membrane bioreactor (MBR) for sewage treatment. Water Sci Techno 2012; 65: 380-392. http://dx.doi.org/10.2166/wst.2012.861

\section{DOI: http://dx.doi.org/10.6000/1929-6037.2015.04.02.5}

(C) 2015 Morita et al.; Licensee Lifescience Global.

This is an open access article licensed under the terms of the Creative Commons Attribution Non-Commercial License (http://creativecommons.org/licenses/by-nc/3.0/) which permits unrestricted, non-commercial use, distribution and reproduction in any medium, provided the work is properly cited. 